\title{
Small molecule targeting of the actin associating protein tropomyosin Tpm3.1 increases neuroblastoma cell response to Rac inhibition of multicellular invasion
}

\author{
Camilla B. Mitchell ${ }^{1, *}$, Justine R. Stehn ${ }^{2,3}$ and Geraldine M. O’Neill ${ }^{1,4}$ \\ ${ }^{1}$ Children's Cancer Research Unit, Kids Research Institute, The Children's Hospital at Westmead, \\ Westmead, New South Wales, Australia, ${ }^{2}$ Novogen Pty Ltd, Hornsby, NSW, Australia, ${ }^{3}$ School of \\ Medical Sciences, University of New South Wales Australia, Sydney, NSW, Australia; and ${ }^{4}$ Discipline of \\ Paediatrics and Child Health, The University of Sydney, Sydney, New South Wales, Australia. \\ *Present address: Division of Cancer Research, Peter MacCallum Cancer Centre, Melbourne, Victoria, \\ Australia
}

Running title: Tpm3.1 and collective invasion.

Keywords: neuroblastoma; invasion; 3-dimensional; spheroid; GTPase

Financial support: This work was generously supported by a Childhood Cancer Cytoskeleton Consortium (C4) Fellowship from The Kids' Cancer Project to C.M.

Corresponding Author: Geraldine M. O’Neill

Children's Cancer Research Unit

Kids Research Institute

The Children's Hospital at Westmead

Locked Bag 4001, Westmead, 2145

This is the author manuscript accepted for publication and has undergone full peer review but has not been through the copy may lead to differences between this version and the Version of Record. Please cite this article as doi: 10.1002/cm.21452

Email: geraldine.oneill@ health.nsw.gov.au 


\begin{abstract}
The migration and invasion of cells through tissues in the body is facilitated by a dynamic actin cytoskeleton. The actin-associating protein, tropomyosin Tpm3.1 has emerged to play important roles in cell migration and invasion. To date, investigations have focused on single cell migration and invasion where Tpm3.1 expression is inversely associated with Rac GTPase-mediated cell invasion. While single cell and collective cell invasion have many features in common, collective invasion is additionally impacted by cell-cell adhesion, and the role of Tpm3.1 in collective invasion has not been established. In the present study we have modelled multicellular invasion using neuroblastoma spheroids embedded in 3D collagen and analysed the function of Tpm3.1 using recently established compounds that target the Tpm3.1 C-terminus. The major findings from our study reveal that combined Rac inhibition and Tpm3.1 targeting result in greater inhibition of multicellular invasion than either treatment alone. Together, the data suggest that Tpm3.1 disruption sensitizes neuroblastoma cells to Rac inhibition of multicellular invasion.
\end{abstract}




\section{Introduction}

When invading through complex 3D tissue environments, cells respond to physical and biochemical cues in the extra-cellular environment facilitating their passage. Responding to changes in the external tissue structure, cells adopt alternative invasion mechanisms which can lead to discrete functional outcomes [Friedl et al., 2012]. Invasive cancer cells have co-opted many of these invasion mechanisms and thus can display a range of individual and multicellular/collective modes of invasion. The actin-associating protein Tpm3.1 (formerly known as Tm5NM1 [Geeves et al., 2015]) is an important player in cell migration and invasion, through regulating the dynamic reorganisation of the actin cytoskeleton and associated focal adhesions [Lees et al., 2011a]. However, while previous studies have addressed Tpm3.1's role in single cell migration and invasion, the role of Tpm3.1 in collective cell invasion modes is not known. This is of particular interest in light of the proposed new role for Tpm3.1 in maintaining the integrity of cell-cell junctions [Caldwell et al., 2014], thus suggesting that Tpm3.1 may differentially affect collective versus single cell invasion. In the present study the role of Tpm3.1 in collective invasion is investigated using multicellular spheroids of neuroblastoma cells embedded in 3D hydrogels combined with small molecule inhibitors of Tpm3.1.

Collectively invading cells variously display sheets, strands, or small clumps of cells invading en masse [Friedl et al., 2012]. In addition to the influence of direct cell-cell adhesion, cells can cooperatively invade with one population influencing the invasion of a second population of cells in a heterogeneous mix of cells (for example [Mitchell and O'Neill, 2016]). Tpm3.1 function in single cell migration and invasion has previously been demonstrated in B35 neuroblastoma cells [Bryce et al., 2003; Bach et al., 2009; Lees et al., 2011b]. Neuroblastomas are a class of paediatric solid tumour that are derived from neural crest cells with a diverse range of single and multicellular invasion patterns [Theveneau and Mayor, 2012]. Reflecting their cellular derivation, patient-derived neuroblastomas exhibit collective invasion into local tissue in orthotopic mouse xenografts [Braekeveldt et al., 2015]. Moreover, multicellular neuroblastoma spheroids embedded in 3D collagen display a range of cooperative and collective invasion behaviours [Mitchell and O'Neill, 2016; Mitchell and O'Neill, 2017].

There are over 40 tropomyosin isoforms generated through differential transcript splicing from 4 coding genes [Gunning et al., 2008]. Their major function is to regulate the dynamics of the associated actin filament, via regulating access of different myosin motors [Clayton et al., 2015; Manstein and Mulvihill, 2016; Bryce et al., 2003; Schevzov et al., 2005]. Tpm3.1 stabilises actin filaments, through myosin II recruitment [Bryce et al., 2003; Schevzov et al., 2005; Clayton et al., 2015; Gateva et al., 2017] and this stabilising function underpins the multiplicity of roles that have been ascribed to this ubiquitously 
expressed protein [Bryce et al., 2003; Bach et al., 2009; Bach et al., 2014; Schevzov et al., 2005; Lim et al., 2015; Clayton et al., 2014; Caldwell et al., 2014; Vlahovich et al., 2009; Lees et al., 2011b; Lees et al., 2013]. Elevated Tpm3.1 expression inhibits both 2-dimensional (2D) and 3D single cell migration [Bryce et al., 2003; Bach et al., 2009; Bach et al., 2014; Lees et al., 2011b]. Conversely, the loss of Tpm3.1 expression, either through genetic deletion or siRNA-mediated depletion, is inversely related to Rac GTPase activity and thus stimulates Rac-dependent, elongated mesenchymal invasion in single invasive cells [Lees et al., 2011b; Lees et al., 2013]. Given the recently described role for Tpm3.1 in cell-cell adhesion [Caldwell et al., 2014], Tpm3.1 may have additional roles to play in collective invasion.

Recently, the Tpm3.1 targeting small molecule TR100, was reported [Stehn et al., 2013]. Subsequent screening for second generation anti-tropomyosin (ATM) drugs that target the Tpm3.1 C-terminus identified a novel compound, ATM-3507, that binds to and impairs Tpm3.1 function [Currier et al., 2017]. In the present study ATM-3507 has been employed in order to investigate the role of Tpm3.1 in multicellular invasion. The results reveal that ATM-3507 induces loss of filamentous actin and switches the invasive morphologies of both single and collectively invading cells. When combined with Rac inhibition, Tpm3.1 targeting decreases multicellular invasion of neuroblastoma spheroids.

\section{Results}

\section{MYCN and non-MYCN neuroblastoma lines are sensitive to ATM3507}

The MYCN gene is an established prognostic indicator for neuroblastoma [Brodeur, 2003] and since MYCN amplification is inversely related to Rac GTPase activity [Mitchell and O'Neill, 2017] we compared Tpm3.1 expression relative to MYCN amplification status in a panel of neuroblastoma cell lines. As previously reported [Stehn et al., 2013], Tpm3.1 expression was similar between representative examples of both non-MYCN amplified, high Rac activity, neuroblastoma (SHEP, SKNAS, SKNSH) and amplified MYCN/low Rac expression (BE2C, IMR32 and CHP134) [Mitchell and O'Neill, 2017] (Figure 1A). ATM-3507 cytotoxicity was next compared between the non-MYCN amplified line SKNSH and MYCN amplified BE2C cells. ATM-3507 had comparable effects on cell viability in both lines, with IC50 values of $5.4 \mu \mathrm{M} \pm 0.5$ and $5.3 \mu \mathrm{M} \pm 0.2$ for SKNSH and BE2C, respectively (Figure 1B).

Based on reports that many drugs are less effective in 3D when compared with $2 \mathrm{D}$ cultures due to cellcell and cell-extracellular matrix interactions [Correia and Bissell, 2012; Krishnan et al., 2016], we next analysed the effect of ATM-3507 on SKNSH and BE2C spheroids embedded in 3D collagen gels. Conditions were standardised to generate spheroids of $500 \mu \mathrm{m}$ maximal diameter, as this size creates an 
optimal physiological gradient of exposure to nutrients [Ivanov et al., 2014]. Subsequent embedding in collagen facilitates analysis of cell invasion, as the collagen matrix provides a biologically active mimetic of the in vivo tissue organisation. Confirming growth in the $3 \mathrm{D}$ cultures, spheroids enlarged over the time course (Figure 1C, compare 0 and 48 hours). Strikingly, incubation with $10 \mu \mathrm{M}$ ATM-3507 ( double the $\mathrm{IC}_{50}$ in $2 \mathrm{D}$ culture) did not alter overall spheroid growth in 3D (Figure 1C), confirmed by quantification of spheroid area (Figure 1D). By contrast, there were less than $10 \%$ viable cells remaining in $2 \mathrm{D}$ cultures treated with $10 \mu \mathrm{M}$ ATM-3507 (Figure 1B). Thus agreeing with other reports in the literature, we find that ATM-3507 effects on cell growth are much reduced in 3D compared with 2D cultures.

\section{ATM-3507 disrupts F-actin detection and induces altered invasive morphologies}

We then further assessed the effect of ATM-3507 in collagen-embedded multicellular SKNSH and BE2C spheroids. Strikingly, ATM-3507 abolished F-actin staining in embedded spheroids of both cell types (Figure 2A). To confirm that this did not simply represent cell loss, cells were labelled with fluorescent cell tracker prior to spheroid formation and embedding. This revealed cell tracker-positive cells lacking phalloidin-staining and thus confirmed that the lack of F-actin detection was not due to cell loss (Figure 2B). Furthermore, the same absence of F-actin detection was observed in single cell suspensions of SKNSH and BE2C cells in 3D collagen (Figure 2C). Assessment of F-actin staining in a dilution series of ATM-3507 $(0,0.4,1.0,4.0$ and $10 \mu \mathrm{M})$ revealed that loss of phalloidin positive cell staining is seen at the highest concentration of ATM-3507 tested (Figure 2D).

Next, we analysed the effect of ATM-3507 on spheroid growth and invasion. As previously established, the SKNSH spheroids displayed both multicellular strand invasion and elongated single cell invasion representing the mixed lineage of this cell line [Mitchell and O'Neill, 2016]. Despite reduced detection of F-actin, spheroid sizes were not significantly different in ATM-3507 treated conditions (Figure 3A). However, the single elongated invasive cells seen under control conditions (Figure 3B, arrows), were absent in ATM-3507-treated spheroids. Instead single dispersed cells had a rounded appearance (Figure 3B, arrow heads). This morphological change was further confirmed through analysis of single cell suspensions of SKNSH cells. Control cultures revealed three distinct morphologies: phalloidin and cell tracker positive elongated cells (Figure 3D), phalloidin and cell tracker positive rounded cells (Figure 3D, asterisks) and phalloidin negative but cell tracker positive rounded cells (Figure 3D arrows). In the presence of ATM-3507 the proportion of elongated cells decreased and the phalloidin negative, celltracker positive round cells predominated. 
Contrasting the invasive behaviour of SKNSH spheroids, the BE2C spheroids displayed a collective sheet of invading cells (Figure 4A), confirmed by time-lapse imaging (Figure 4B). The invading sheet has a cobblestone F-actin appearance, reflecting adhesion between neighbouring cells in the invading sheet (Figure 4A, enlarged image and see additional example in Supplementary Figure 2). At early time points cells at the spheroid periphery extended long, thin membrane processes into the collagen (Figure 4B, 0-8 hours). Subsequently, a sheet of cells emerged from the spheroid and continued to expand and migrate into the collagen matrix (Figure 4B, 16 hours, arrow). Occasional individual cells were seen to escape the advancing front (Figure 4B arrow heads). Exposure to ATM-3507 did not affect total BE2C spheroid size (Figure 3A). However, ATM-3507 induced the appearance of single cells at the spheroid periphery (Figure 3C), suggesting that ATM-3507 may induce the loss of cell-cell adhesion. Agreeing with this, individual cells unattached to their neighbours are seen in the bright field images of BE2C spheroids treated with ATM-3507 (Figure 1C). This was further supported by analysis of single cell suspensions of BE2C cells which, despite efforts to disaggregate into single cell suspensions prior to embedding, exhibit small clusters of cells (Figure 3E). These small clusters of adherent cells are lost when cultured in the presence of ATM-3507 (best seen in the Hoescht images, Figure 3E). Collectively, the data reveal that ATM-3507 induced switches in the invasive modes of both SKNSH and BE2C cells. As spheroid sizes were not significantly changed in the presence of ATM-3507, this suggests that despite the changes to invasive morphology, the cells retained the same proliferative and invasive competency as under control conditions.

\section{Tpm3.1 targeting increases response to Rac inhibition}

We next considered whether ATM-3507 might induce changes in the cytoskeleton that could prime the cells for response to other agents that affect the cytoskeleton. Given that Tpm3.1 expression is inversely related to Rac activity, response to Rac inhibition versus Rac inhibition combined with ATM-3507 was next assessed. Spheroids were treated with ATM-3507 and the potent Rac inhibitor EHT1864 alone or in combination. Importantly, combined treatment with ATM-3507 and EHT1864 resulted in smaller SKNSH and BE2C spheroids than either compound alone (Figure 5A). Moreover, there was a striking reduction in the appearance of individual elongated invasive cells in the SKNSH cultures. Quantification confirmed significant smoothing of spheroid boundaries (Figure 5B). Collectively, these data suggest that ATM-3507 increases SKNSH and BE2C spheroid response to Rac inhibition, and together the compounds inhibit both growth and invasion. 
An important question was whether the sensitisation to Rac inhibition was secondary to the loss of F-actin or whether this was a primary effect of the loss of Tpm3.1 function. Among the second generation compounds targeting the Tpm3.1 C-terminus generated were two additional compounds, ATM-1001 and ATM-4015. Recently, ATM-1001 has been demonstrated to have a similar mode of action to ATM-3507 [Kee et al., 2018]. Neither of these compounds induced the loss of phalloidin staining (Figure 6A). Cytotoxicity quantification revealed that both BE2C and SKNSH cells were sensitive to ATM-1001 (IC50 $=10.1 \mu \mathrm{M} \pm 2.5$ and $9.1 \mu \mathrm{M} \pm 1.0$, respectively) and ATM-4015 (IC50 $=7.0 \mu \mathrm{M} \pm 1.1$ and $9.3 \mu \mathrm{M}$ \pm 2.0 , respectively). Importantly, either ATM-1001 or ATM-4015 in combination with Rac inhibition significantly reduced SKNSH spheroid size and inhibited the invasion of single elongated invasive cells (Figure 6A and 6C). In contrast, neither ATM-1001 nor ATM-4015 in combination with Rac inhibition significantly decreased the BE2C spheroid size relative to reduced size seen with Rac inhibition alone (Figure 6B and 6D). However, combined treatment did induce a smoothed BE2C spheroid boundary indicating that this combination treatment significantly inhibited invasion (Figure 6E). Collectively, these data suggest that Tpm3.1 targeting sensitizes cells to Rac-inhibitor mediated inhibition of cell invasion.

Finally, it was noted that following exposure of BE2C spheroids to either ATM-1001 or ATM-4015 there was no evidence of loss of cell-cell adhesion at the spheroid peripheries (Figure 6B). Collectively, these data suggest that the loss of cell-cell adhesion induced by ATM-3507 is secondary to the loss of F-actin induced by this compound.

\section{Discussion}

Since Tpm3.1 depletion can induce Rac activity and increase cell migration [Lees et al., 2013] it was important to establish whether molecules targeting Tpm3.1 might have the unintended consequence of inducing invasion. The present study has revealed that Tpm3.1 targeting drugs do not induce invasion. Moreover, we demonstrate that ATM-3507 increases cellular response to Rac inhibition of collective cell invasion. Based on the data, we propose that anti-Tpm3.1 mediated changes in the actin cytoskeletal network increases the inhibitory effects of the major actin regulator Rac GTPase.

Based on reports that mouse embryo fibroblasts lacking Tpm3.1 display elongated mesenchymal invasion [Lees et al., 2013], it was predicted that anti-Tpm3.1 compounds may induce morphological transition to mesenchymal invasion. Surprisingly, neither anti-Tpm compound ATM-1001 nor ATM-4015 altered cellular morphologies, raising the question why the anticipated mesenchymal transition was not observed. The answer may be found in recent analyses which have revealed that while (TR100) perturbs Tpm3.1 
regulated actin filament depolymerisation [Bonello et al., 2016], Tpm3.1 still associates with the actin filaments in the presence of the drug. This therefore may explain why anti-Tpm3.1 drugs do not phenocopy all effects of genetic deletion. Tpm3.1 recruits myosin II motors to actin filaments [Bryce et al., 2003; Schevzov et al., 2005; Clayton et al., 2015; Gateva et al., 2017] and elongated mesenchymal invasion requires inhibition of myosin II motor activity at the cell's leading edge [Asokan et al., 2014]. Thus, continued association of Tpm3.1 with actin filaments following exposure to anti-Tpm3.1 drugs may maintain myosin II activity at the leading edge and thereby block induction of mesenchymal invasion. Despite this, anti-Tpm3.1 drugs appear to prime the cells for further insults to the actin cytoskeleton network, possibly through increased activation of Rac GTPase [Lees et al., 2013], since the addition of Rac inhibitors tips the cells into rounded morphologies.

The demonstration that neither ATM-1001 nor ATM-4015 alone affected growth and invasion of neuroblastoma spheroids is in contrast to previous studies where TR100 inhibited growth and invasion of melanoma spheroids [Stehn et al., 2013]. This may reflect cancer type differences (melanoma versus neuroblastoma). However, the earlier study also showed that TR100 inhibited migration of the SHEP neuroblastoma cell line. These migration analyses were performed under $2 \mathrm{D}$ conditions and so are not directly comparable to the 3D assays in the present study [Bradbury et al., 2012]. Despite this, the different invasion phenotypes exhibited by SHEP, SKNSH and BE2C spheroids in 3D cultures may be relevant. SHEP spheroids display Rac-dependent single cell invasion [Mitchell and O'Neill, 2017], SKNSH have a mix of single cell and collective strand invasion [Mitchell and O'Neill, 2016] and BE2C exhibit collective sheet invasion (this study). Therefore different responses to drug treatments may reflect differences between collective and single cell invasion. Supporting this suggestion, under the same collagen gel assay conditions used in the present study, cells from melanoma spheroids use a single cell invasion mode [Haeger et al., 2014]. Increased collagen density reportedly switches melanoma cells to collective invasion [Haeger et al., 2014]. In future it would be interesting to test whether melanoma spheroids induced to undergo collective invasion respond differently to anti-Tpm3.1 drugs.

Of the three anti-Tpm3.1 compounds tested in the present study, ATM-3507 caused the most striking response, inducing loss of phalloidin staining from both single cell cultures and multicellular spheroids. This suggested that ATM-3507 may be stimulating F-actin disassembly and further that it is more efficient at destabilising actin filaments than either of the other two compounds. However, the present data cannot rule out that ATM-3507 has induced structural change to the actin filament that prevents phalloidin from binding to the filament. It was noted that ATM-3507 treatment induces dispersal of the 
BE2C cells at the spheroid boundary into single cells. Recent reports indicate that Tpm3.1 mediates junctional tension at cell-cell adhesions [Caldwell et al., 2014]. Therefore, ATM-3507 may induce loss of cell-cell adhesion between collectively invading cells, thereby switching cells to single cell, Racdependent invasion. We propose that the anti-Tpm3.1-induced switch from a collective to single cell invasion mode correspondingly increases the cells to Rac inhibition. Importantly, single versus collective cell invasion may predispose to blood versus lymph-based dissemination, respectively [Giampieri et al., 2009]. In summary, data presented in this study reveal that Tpm3.1 targeting can expose new vulnerabilities in the actin cytoskeleton that increase response to the inhibition of other actin regulators.

\section{Materials and Methods}

\section{Cell lines and cell culture}

Cultured neuroblastoma cell lines (SHEP, SK-N-AS, SKNSH, BE2C, IMR32 and CHP134) were kindly provided by Dr. Loretta Lau (Kids Research Institute, Sydney, Australia). SK-N-AS, SKNSH, BE2C, and CHP134 cell lines were obtained mid-2014 and early 2015 and were not further authenticated after having been received from Dr Lau, who purchased the lines from EACC. SHEP and IMR32 were from previous laboratory stocks [Yager et al., 2003] and have not been further authenticated. CHP134 cells were maintained in RPMI-1640 medium supplemented with 10\% FBS. All other cell lines were maintained in Dulbecco's modified eagle's medium (DMEM) supplemented with 10\% FBS. Preparation of single cell suspensions and spheroid embedding in collagen gels were performed as previously described [Mitchell and O'Neill, 2017]. Spheroids are viewed by bright field microscopy regularly throughout the experiments to ensure that under control conditions spheroids continue active growth and invasion. Collagen gels containing cells and spheroids were allowed to polymerise for 1 hour at $37^{\circ} \mathrm{C}$, subsequently complete media with or without pharmacological agents was added and all cultures were incubated for a further 48 hours. Custom-designed Rac1 siRNAs from Invitrogen and conditions for use have been previously described [Mitchell and O'Neill, 2017]. Cells were transfected with siRNA prior to suspension in collagen.

\section{Drugs and inhibitors}


Anti-tropomyosin (ATM) drugs targeting the C-terminal end of Tpm3.1, were provided by Novogen and have been previously described [Currier et al., 2017; Heaton, 2016; James, 2015; James, 2016; Kee et al., 2018]. Stock solutions (10mM) were prepared in DMSO. Final concentration of drug used in experiments was $10 \mu \mathrm{M}$ for ATM compounds and $25 \mu \mathrm{M}$ for EHT 1864 (Tocris Bioscience).

\section{Cytotoxicity assays}

Cells plated in 96 well dishes were treated with ATM compounds and viability measured after 72 hours using MTS (3-(4,5-dimethylthiazol-2-yl)-5-(3-carboxymethoxyphenyl)-2-(4-sulfophenyl)-2Htetrazolium). Percentage viability was calculated relative to vehicle control-treated cells. IC50 (inhibitory concentration at which 50\% cell are non-viable) was determined by non-linear regression sigmoidal doseresponse (variable slope) curve fitting, using GraphPad Prism V6.05.

\section{Western blotting, immunofluorescence and imaging}

Proteins were extracted as previously described [Bach et al., 2014]. Protein concentrations were equalized following quantification with the Biocinchoninic acid (BCA) Protein Assay Kit (Pierce Biotechnology). SDS-PAGE and immunoblotting were performed using standard conditions and 10\% SDSpolyacrylamide gels. Tropomyosin Tpm3.1 was detected using a sheep polyclonal anti- $\gamma 9 \mathrm{~d}$ antibody kindly provided by Prof Peter Gunning. Immunofluorescence of embedded cells and spheroids was performed as previously described [Mitchell and O'Neill, 2016]. Confocal imaging of fixed and immunostained cells and spheroids was performed on a Leica SP5 II confocal microscope with a $10 \mathrm{x}$ air objective. Live cell time-lapse imaging was performed using an ORCA ERG cooled CCD camera (Hamamatsu, SDR Clinical Technology) and a 20x air objective on an Olympus IX81 inverted microscope equipped with an environmental heat chamber maintained at $37^{\circ} \mathrm{C}$ and operated by Metamorph V6.3 software (Molecular Devices).

\section{Image analysis}

Morphology analysis of single cell suspensions was performed as previously described [Mitchell and O'Neill, 2017]. Quantification of spheroids was achieved using Metamorph V7.7 to determine the equivalent radius (the radius of a circle that would contain an area equal to that of the object) of thresholded maximum projections of phalloidin-stained spheroids. To account for variation between biological replicates, each set of radii were expressed relative to the matched control untreated spheroid for that replicate series. Due to loss of phalloidin-staining following treatment with ATM-3507 compound, Hoescht blue images of spheroids treated with this compound were instead analysed using Metamorph V7.1 to measure the shape factor $\left(4 \pi \mathrm{A} / \mathrm{P}^{2}\right.$, where $\mathrm{A}=$ area and $\mathrm{P}=$ perimeter $)$. Values of 1.0 
indicate a perfect circle and thus higher values reflect a smooth spheroid edge and reduced cell invasion. All error bars on graphs show SEM. All final colour adjustments of fluorescence micrographs were made in Adobe Photoshop.

\section{Acknowledgements}

This work was supported by a Childhood Cancer Cytoskeleton Consortium (C4) Fellowship from the Kids' Cancer Project to C.M. We gratefully acknowledge Laurence Cantrill for assistance with imaging. We thank Kazia Therapeutics Ltd (formerly Novogen Ltd) for the supply of anti-tropomyosin compounds.

\section{Conflicts of interest}

C.M. and G.M.O’N declare no conflicts of interest. J.R.S was an employee of Novogen Pty Ltd, a company which is commercialising anti-tropomyosin drug technology. 


\section{References}

Asokan SB, Johnson HE, Rahman A, King SJ, Rotty JD, Lebedeva IP, Haugh JM and Bear JE. 2014. Mesenchymal chemotaxis requires selective inactivation of myosin II at the leading edge via a noncanonical PLCgamma/PKCalpha pathway. Dev Cell 31(6):747-60.

Bach CT, Murray RZ, Owen D, Gaus K and O'Neill GM. 2014. Tropomyosin Tm5NM1 spatially restricts src kinase activity through perturbation of Rab11 vesicle trafficking. Mol Cell Biol 34(24):4436-46.

Bach CT, Creed S, Zhong J, Mahmassani M, Schevzov G, Stehn J, Cowell LN, Naumanen P, Lappalainen P, Gunning PW et al. 2009. Tropomyosin isoform expression regulates the transition of adhesions to determine cell speed and direction. MolCell Biol 29(6):1506-14.

Bonello TT, Janco M, Hook J, Byun A, Appaduray M, Dedova I, Hitchcock-DeGregori S, Hardeman EC, Stehn JR, Bocking T et al. 2016. A small molecule inhibitor of tropomyosin dissociates actin binding from tropomyosin-directed regulation of actin dynamics. Sci Rep 6:19816.

Bradbury P, Fabry B and O'Neill GM. 2012. Occupy tissue: The movement in cancer metastasis. Cell Adh Migr 6(5):424-32.

Braekeveldt N, Wigerup C, Gisselsson D, Mohlin S, Merselius M, Beckman S, Jonson T, Borjesson A, Backman T, Tadeo I et al. 2015. Neuroblastoma patient-derived orthotopic xenografts retain metastatic patterns and geno- and phenotypes of patient tumours. Int J Cancer 136(5):E252-61. 
Brodeur GM. 2003. Neuroblastoma: biological insights into a clinical enigma. Nat Rev Cancer 3(3):203-

16.

Bryce NS, Schevzov G, Ferguson V, Percival JM, Lin JJ, Matsumura F, Bamburg JR, Jeffrey PL, Hardeman EC, Gunning P et al. 2003. Specification of actin filament function and molecular composition by tropomyosin isoforms. Mol Biol Cell 14(3):1002-16.

Caldwell BJ, Lucas C, Kee AJ, Gaus K, Gunning PW, Hardeman EC, Yap AS and Gomez GA. 2014.

Tropomyosin isoforms support actomyosin biogenesis to generate contractile tension at the epithelial zonula adherens. Cytoskeleton (Hoboken, NJ) 71(12):663-76.

Clayton JE, Pollard LW, Murray GG and Lord M. 2015. Myosin motor isoforms direct specification of actomyosin function by tropomyosins. Cytoskeleton (Hoboken, NJ) 72(3):131-45.

Clayton JE, Pollard LW, Sckolnick M, Bookwalter CS, Hodges AR, Trybus KM and Lord M. 2014. Fission yeast tropomyosin specifies directed transport of myosin-V along actin cables. Mol Biol Cell 25(1):66-75.

Correia AL and Bissell MJ. 2012. The tumor microenvironment is a dominant force in multidrug resistance. Drug resistance updates : reviews and commentaries in antimicrobial and anticancer chemotherapy 15(1-2):39-49.

Currier MA, Stehn JR, Swain A, Chen D, Hook J, Eiffe E, Heaton A, Brown D, Nartker BA, Eaves DW et al. 2017. Identification of Cancer-Targeted Tropomyosin Inhibitors and Their Synergy with Microtubule Drugs. Mol Cancer Ther 16(8):1555-65.

This article is protected by copyright. All rights reserved. 
Friedl P, Locker J, Sahai E and Segall JE. 2012. Classifying collective cancer cell invasion. Nat Cell Biol 14(8):777-83.

Gateva G, Kremneva E, Reindl T, Kotila T, Kogan K, Gressin L, Gunning PW, Manstein DJ, Michelot A and Lappalainen P. 2017. Tropomyosin Isoforms Specify Functionally Distinct Actin Filament Populations In Vitro. Curr Biol 27(5):705-13.

Geeves MA, Hitchcock-DeGregori SE and Gunning PW. 2015. A systematic nomenclature for mammalian tropomyosin isoforms. J Muscle Res Cell Motility 36(2):147-53.

Giampieri S, Manning C, Hooper S, Jones L, Hill CS and Sahai E. 2009. Localized and reversible TGFbeta signalling switches breast cancer cells from cohesive to single cell motility. Nat Cell Biol 11(11):1287-96.

Gunning P, O'Neill G and Hardeman E. 2008. Tropomyosin-based regulation of the actin cytoskeleton in time and space. Physiol Rev 88(1):1-35.

Haeger A, Krause M, Wolf K and Friedl P. 2014. Cell jamming: collective invasion of mesenchymal tumor cells imposed by tissue confinement. Biochim Biophys Acta 1840(8):2386-95.

Heaton A, Eiffe, E, Pottabathini, N, Gunning, P; NOVOGEN LTD (16-20 Edgeworth David Avenue, Hornsby, New South Wales 2077, AU), assignee. 2016. Functionalised and substituted indoles as anticancer agents patent WO/2016/008011.

This article is protected by copyright. All rights reserved. 
Ivanov DP, Parker TL, Walker DA, Alexander C, Ashford MB, Gellert PR and Garnett MC. 2014.

Multiplexing spheroid volume, resazurin and acid phosphatase viability assays for high-throughput screening of tumour spheroids and stem cell neurospheres. PloS one 9(8):e103817.

James I, Dixon, I, Feutrill, J, Cuzzupe, A, Treutlein, H, Zeng, J., Nero, T; NOVOGEN LTD (16-20 Edgeworth David Avenue, Hornsby, New South Wales 2077, AU), assignee. 2015. Functionalised and substituted indoles as anti-cancer agents patent WO/2015/074123.

James I, Dixon, I, Heaton, A, Eiffe, E, Gunning, P; NOVOGEN LTD (16-20 Edgeworth David Avenue, Hornsby, New South Wales 2077, AU), assignee. 2016. Functionalised and substituted carbazoles as anticancer agents patent WO/2016/008010.

Kee AJ, Chagan J, Chan JY, Bryce NS, Lucas CA, Zeng J, Hook J, Treutlein H, Laybutt DR, Stehn JR et al. 2018. On-target action of anti-tropomyosin drugs regulates glucose metabolism. Sci Rep 8(1):4604.

Krishnan R, Park JA, Seow CY, Lee PV and Stewart AG. 2016. Cellular Biomechanics in Drug Screening and Evaluation: Mechanopharmacology. Trends Pharmacol Sci 37(2):87-100.

Lees JG, Bach CT and O'Neill GM. 2011a. Interior decoration: Tropomyosin in actin dynamics and cell migration. Cell Adh Migr 5(2):181-6.

Lees JG, Bach CTT, Bradbury P, Paul A, Gunning PW and O'Neill GM. 2011b. The actin-associating protein Tm5NM1 blocks mesenchymal motility without transition to amoeboid motility. Oncogene 30(10):124151. 
Lees JG, Ching YW, Adams DH, Bach CT, Samuel MS, Kee AJ, Hardeman EC, Gunning P, Cowin AJ and O'Neill GM. 2013. Tropomyosin regulates cell migration during skin wound healing. J Invest Dermatol 133(5):1330-39.

Lim CY, Bi X, Wu D, Kim JB, Gunning PW, Hong W and Han W. 2015. Tropomodulin3 is a novel Akt2 effector regulating insulin-stimulated GLUT4 exocytosis through cortical actin remodeling. Nat Comms 6:5951.

Manstein DJ and Mulvihill DP. 2016. Tropomyosin-Mediated Regulation of Cytoplasmic Myosins. Traffic 17(8):872-7.

Mitchell CB and O'Neill GM. 2016. Cooperative cell invasion: matrix metalloproteinase-mediated incorporation between cells. Mol Biol Cell 27(21):3284-92.

Mitchell CB and O'Neill GM. 2017. Rac GTPase regulation of 3D invasion in neuroblastomas lacking MYCN amplification. Cell Adh Migr 11(1):68-79.

Schevzov G, Bryce NS, Almonte-Baldonado R, Joya J, Lin JJ, Hardeman E, Weinberger R and Gunning P. 2005. Specific features of neuronal size and shape are regulated by tropomyosin isoforms. Mol Biol Cell 16(7):3425-37.

Stehn JR, Haass NK, Bonello T, Desouza M, Kottyan G, Treutlein H, Zeng J, Nascimento PR, Sequeira VB, Butler TL et al. 2013. A novel class of anticancer compounds targets the actin cytoskeleton in tumor cells. Cancer Res 73(16):5169-82. 
Theveneau E and Mayor R. 2012. Neural crest delamination and migration: from epithelium-tomesenchyme transition to collective cell migration. Dev Biol 366(1):34-54.

Vlahovich N, Kee AJ, Van der Poel C, Kettle E, Hernandez-Deviez D, Lucas C, Lynch GS, Parton RG, Gunning PW and Hardeman EC. 2009. Cytoskeletal tropomyosin Tm5NM1 is required for normal excitation-contraction coupling in skeletal muscle. Mol Biol Cell 20(1):400-9.

Yager M, Hughes J, Lovicu F, Gunning P, Weinberger R and O'Neill G. 2003. Functional analysis of the actin-binding protein, tropomyosin 1, in neuroblastoma. British J Cancer 89(5):860-63. 


\section{Figure Legends}

Figure 1: Tpm3.1 expression in neuroblastoma and treatment with ATM-3507. A. Western blot showing Tpm3.1 expression in the indicated neuroblastoma lines. HSP70 expression is shown to confirm equal protein loading. B. Cell viability of BE2C and SKNSH cells in response to increasing concentrations of ATM-3507 for 72 hours. Data shown are the average of 3 independent repeats. Error bars $=$ SEM. C. Bright-field images at 0 and $48 \mathrm{~h}$ of BE2C and SKNSH spheroids embedded in collagen matrix under control conditions or in the presence of $10 \mu \mathrm{M}$ ATM-3507, as indicated. Scale bar $=500$ $\mu \mathrm{m}$. D. Histogram shows the area of spheroids embedded in collagen and treated as indicated for 48 hours. NS = not significant, Students' $t$ test. $>4$ spheroids analysed per condition.

Figure 2: ATM-3507 induces loss of F-actin in 3D spheroid and single cell suspension cultures. A. Representative confocal images (maximum projections) of SKNSH and BE2C spheroids embedded in collagen gels under control conditions (left hand panels) and exposed to ATM-3507 for $48 \mathrm{~h}$. Spheroids were stained with phalloidin (red) and Hoescht blue (blue). Scale bar $500 \mu \mathrm{m}$. Single Z-slices of the same images are shown in Supplementary Figure 1. B. Representative confocal images (maximum projections) of collagen embedded SKNSH and BE2C spheroids after $48 \mathrm{~h}$ of incubation with $10 \mu \mathrm{M}$ ATM-3507. Prior to embedding, cells were incubated with cell tracker (green). Following incubation and drug treatment spheroids were fixed and counter-stained with phalloidin (red) and Hoescht blue (blue). Scale bar $500 \mu \mathrm{m}$. C. Maximum projections of SKNSH and BE2C cells cultured as single cell suspensions in 3D collagen gels, showing control conditions (DMSO, top panels), and following treatment with $10 \mu \mathrm{M}$ ATM-3507. Cells were labelled with cell tracker (green) prior to embedding in the 3D collagen gels and after $48 \mathrm{~h}$ cells were fixed and counter-stained with phalloidin (red). Scale bar $200 \mu \mathrm{m}$. Insets $=2.4 \mathrm{x}$ magnification. Single Z-slices of the same images are shown in Supplementary Figure 1. D. Cell tracker labelled (green) SKNSH spheroids embedded in 3D collagen gels, treated with increasing concentrations of ATM-3507 and then fixed and counter-stained with phalloidin (red) and Hoescht (blue). Scale bar = $250 \mu \mathrm{m}$.

Figure 3. ATM-3507 induces altered invasive morphologies. A. Maximum projection of SKNSH spheroids embedded in 3D collagen under the indicated conditions. Cells were labelled with cell tracker prior to spheroid formation and embedding. $3.5 \mathrm{x}$ magnifications of the regions boxed in white are shown in the images on the right hand side. Arrows point to elongated cells under control conditions and arrow 
heads point to rounded cells in ATM-3507 treated (48 hours) cultures. B. As for A, except with BE2C cells. Note the dispersed pattern of the cells in the magnified region from the ATM-3507 treated spheroids. Scale bars $=500 \mu \mathrm{m}$. C. Single cell suspensions of SKNSH cells in 3D collagen, labelled with cell tracker (green) prior to embedding. Arrows point to examples of rounded, cell trackerpositive/phalloidin-negative cells. Asterisks indicate rounded, cell tracker-positive/phalloidin-positive cells. D. As for C, except with BE2C cells. Note the close packing of the cell clusters in the control condition that is lost in the ATM-3507 treated cells. In all cases embedded cells were incubated with pharmacological agents for 48 hours. Scale bars $=200 \mu \mathrm{m}$.

Figure 4. BE2C spheroids exhibit collective sheet invasion. A. Confocal images (maximum projections) of spheroids $48 \mathrm{~h}$ after plating and stained with phalloidin (red) and Hoescht blue (blue). Scale bar $=500 \mu \mathrm{m}$. Magnified section highlights morphology of the invasive cell front of the BE2C spheroid. B. Representative montage of time-lapse images of collagen embedded BE2C spheroids over a $24 \mathrm{~h}$ time period. White arrow heads point to individual invasive cells. Arrow points to collectively invading sheet of cells.

Figure 5. Anti-tropomyosin compound ATM-3507 sensitises both SKNSH and BE2C spheroids to Rac inhibition. A. Representative SKNSH and BE2C spheroids treated with EHT1864 and ATM-3507 alone and in combination. Hoescht blue stained spheroids are shown for each condition. Scale bar 500 $\mu \mathrm{m}$. Green outlines around the spheroids show the spheroid boundaries used to determine the shape factors. B. Histograms show the average shape factor of spheroids under the indicated treatment conditions. Data for SKNSH spheroids averaged from at least 5 independent experiments with duplicate spheroids per experiment; data for BE2C spheroids averaged from at least 7 independent experiments with duplicate spheroids per experiment. Error bars equal SEM. $* * * \mathrm{p}<0.01$, one way ANOVA. In all cases embedded spheroids were incubated with pharmacological agents for 48 hours.

\section{Figure 6. Sensitisation to Rac inhibition by Tpm3.1 targeting is not due to F-actin loss.} Representative confocal images (maximum projections) of SKNSH (A) and BE2C (B) spheroids embedded in collagen gels and incubate for $48 \mathrm{~h}$ under the indicated conditions. Spheroids were stained with phalloidin (red) and Hoescht blue (blue). Scale bar $500 \mu \mathrm{m}$. C \& D. Quantification of spheroid sizes under the indicated treatment conditions. E. Average shape factor measured on phalloidin-stained 
spheroids under the indicated treatment conditions. Data are the average of at least 7 individual spheroids per condition from three independent repeats and are normalised to the matched control within each experiment. $* \mathrm{p}<0.05, * * \mathrm{p}<0.01, * * * \mathrm{p}<0.001$ (compared to control values), one-way ANOVA with Tukey's post-comparison test. Error bars equal SEM. In all cases embedded spheroids were incubated with pharmacological agents for 48 hours.

\section{Supplementary Figure 1}

Images of single confocal slices. (A) Single Z-slices from the maximum Z-projection images shown in Figure 2A and (B) Figure 2C.

\section{Supplementary Figure 2}

Images of single confocal slices. Images shown are a single confocal slice of a collagen embedded BE2C spheroid. The grey scale images show F-actin and nuclei staining and the coloured image is a merge of these stains.

\section{Supplementary Figure 3}

Spheroid quantification at time zero. Bright field images of SKNSH and BE2C spheroids immediately post-embedding in 3D collagen (0 hours). The histogram shows spheroid area. NS = not significant, Students' $t$-test. 

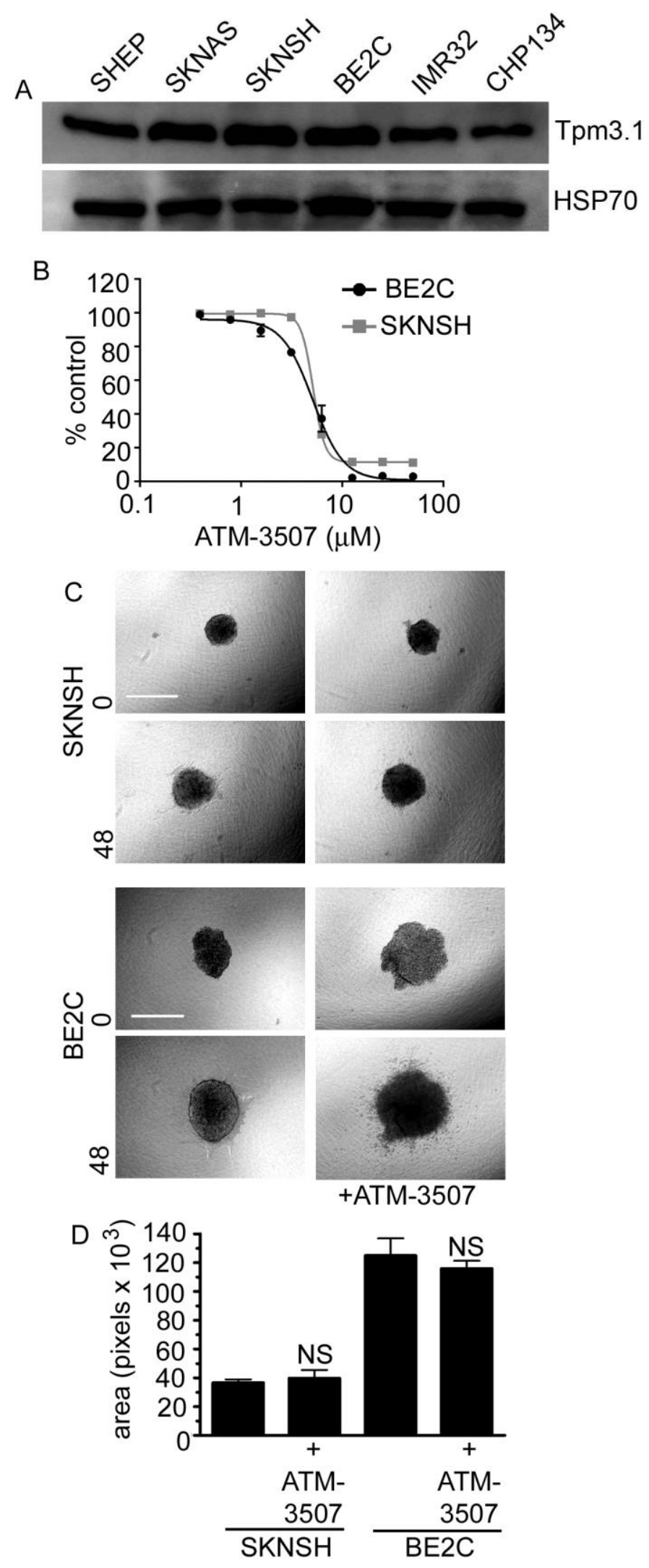

This article is protected by copyright. All rights reserved. 

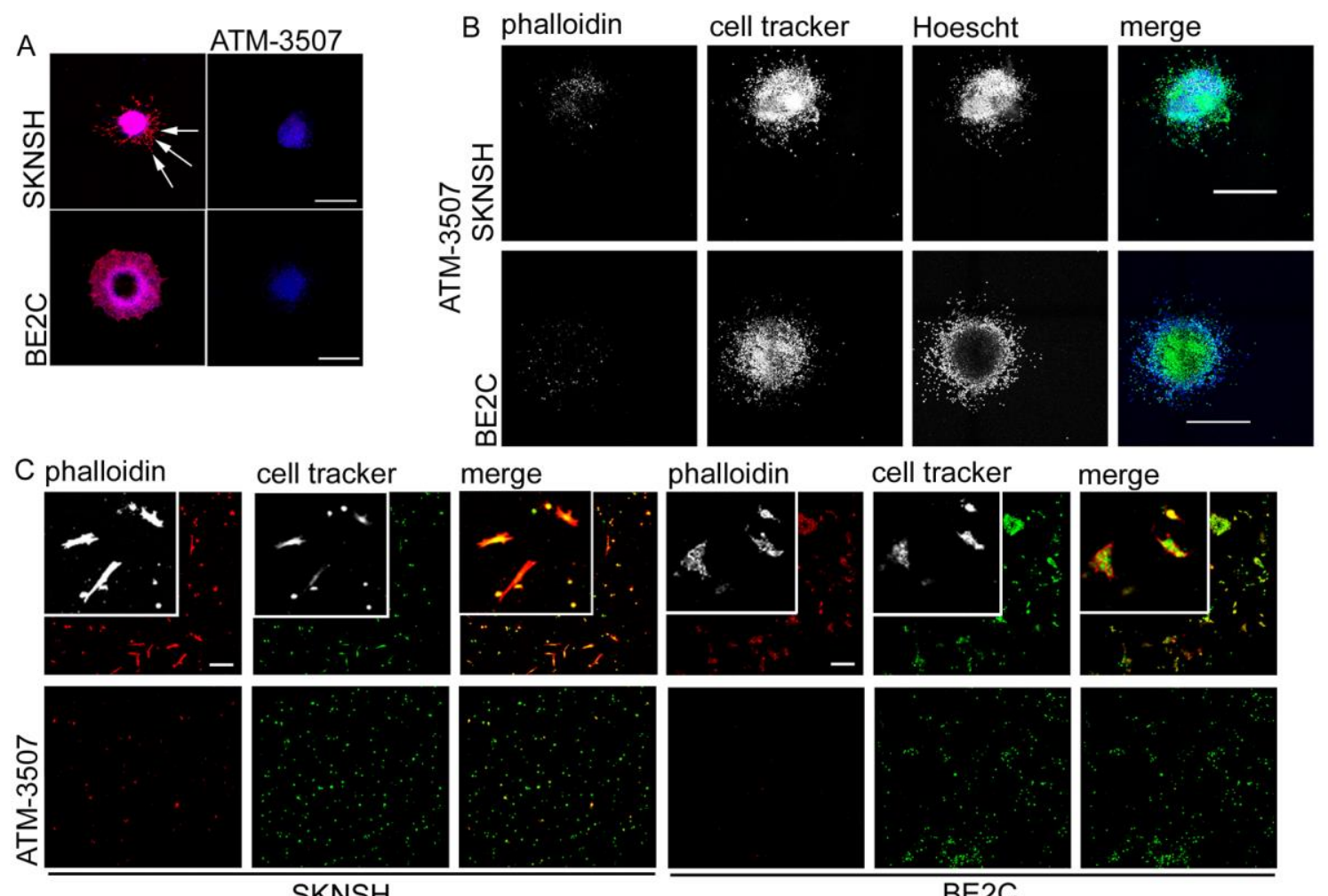

phalloidin

cell tracker

merge
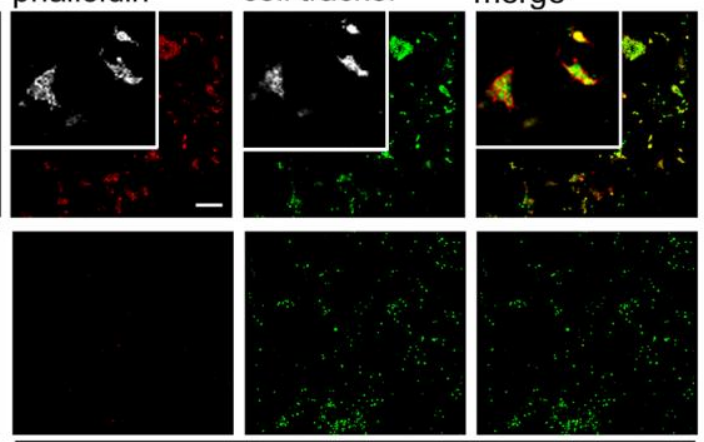

SKNSH
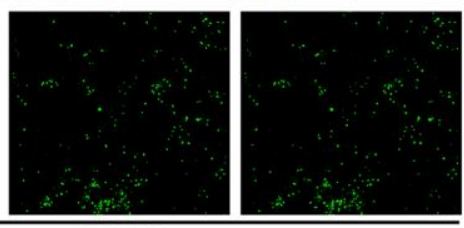

BE2C

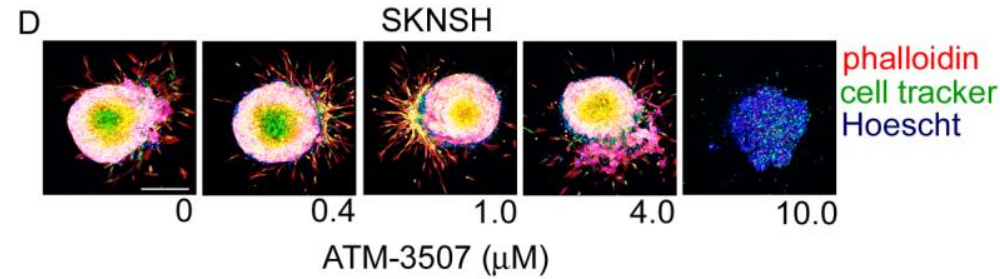




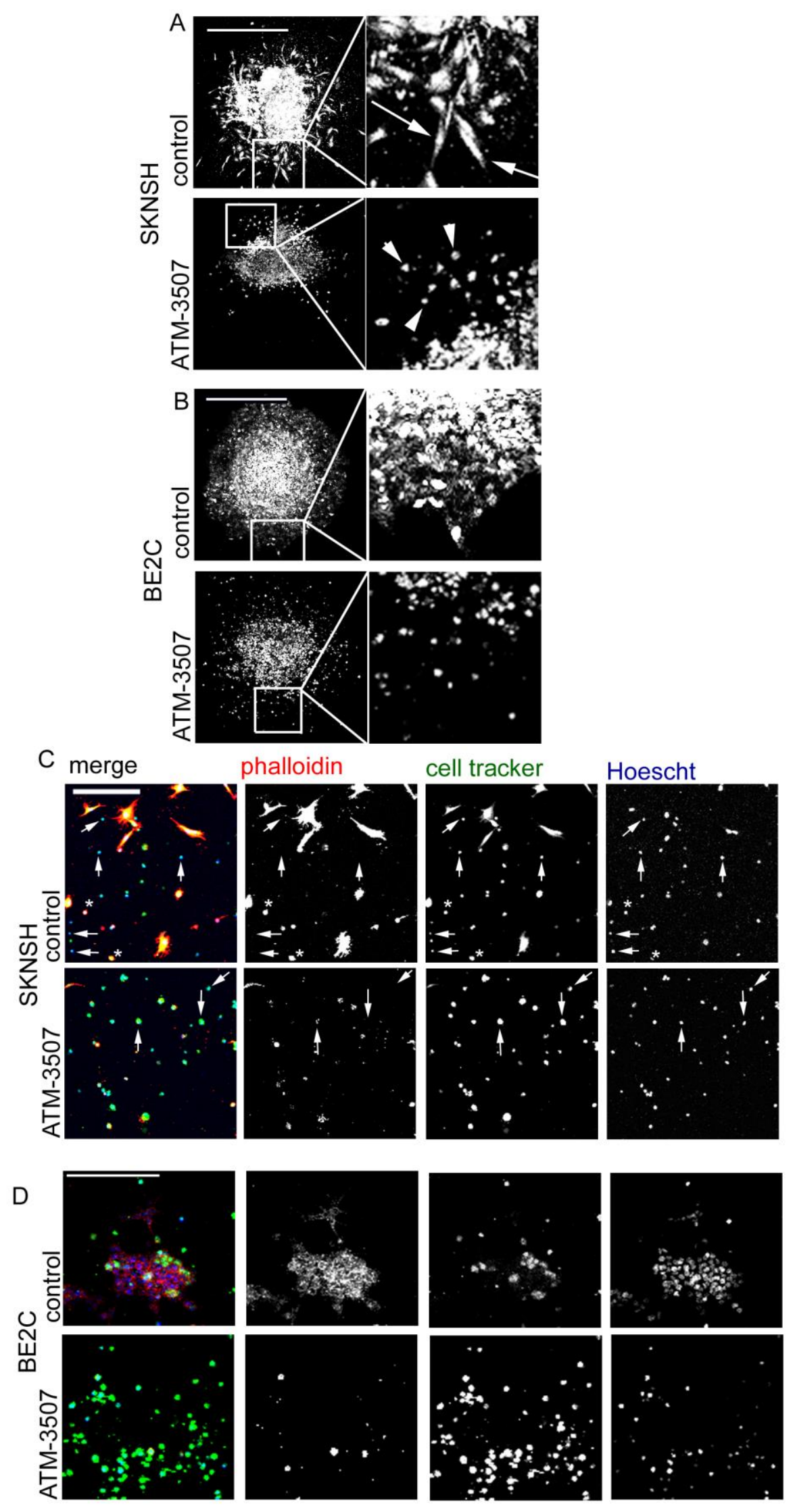

This article is protected by copyright. All rights reserved. 

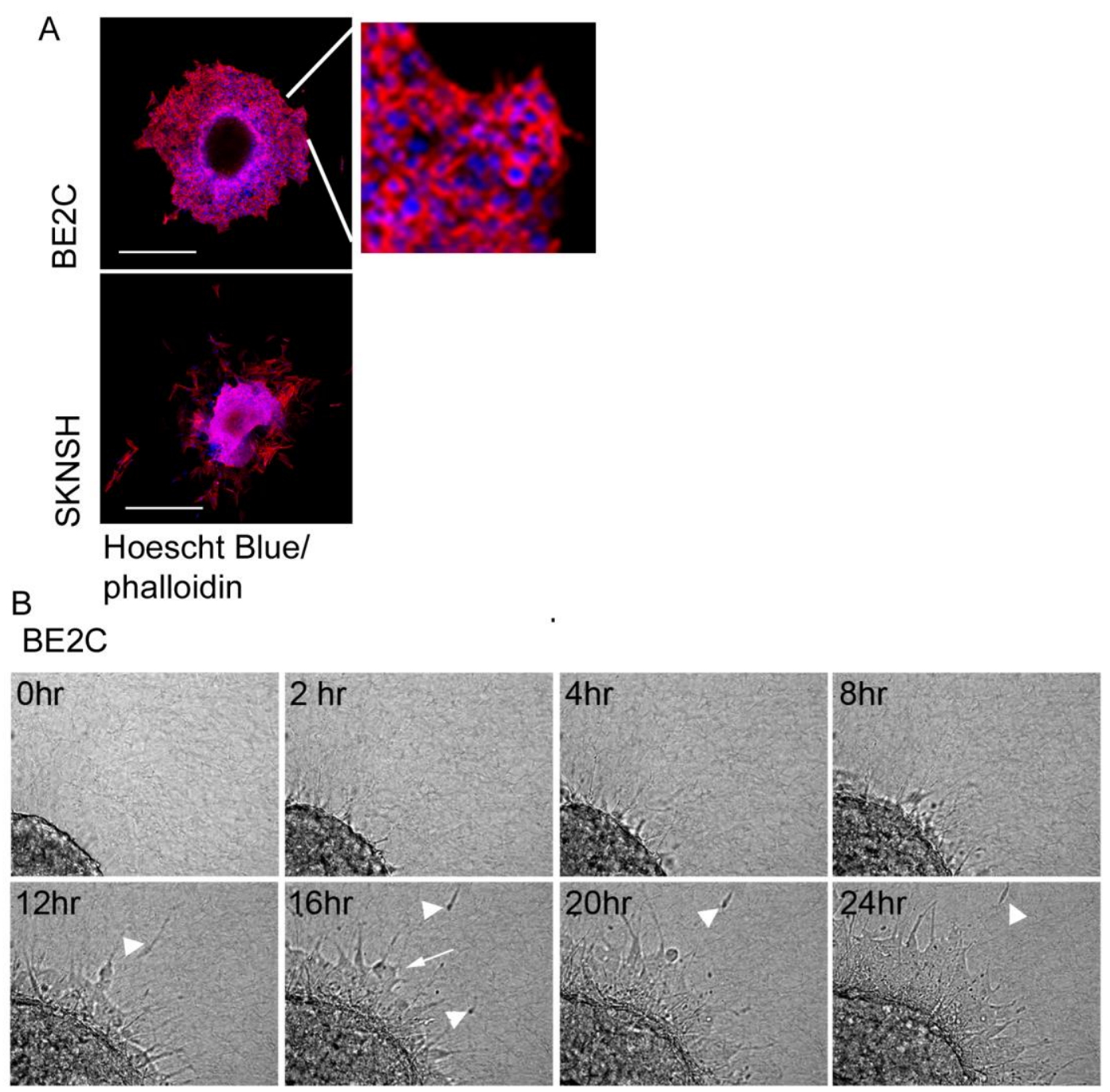

This article is protected by copyright. All rights reserved. 


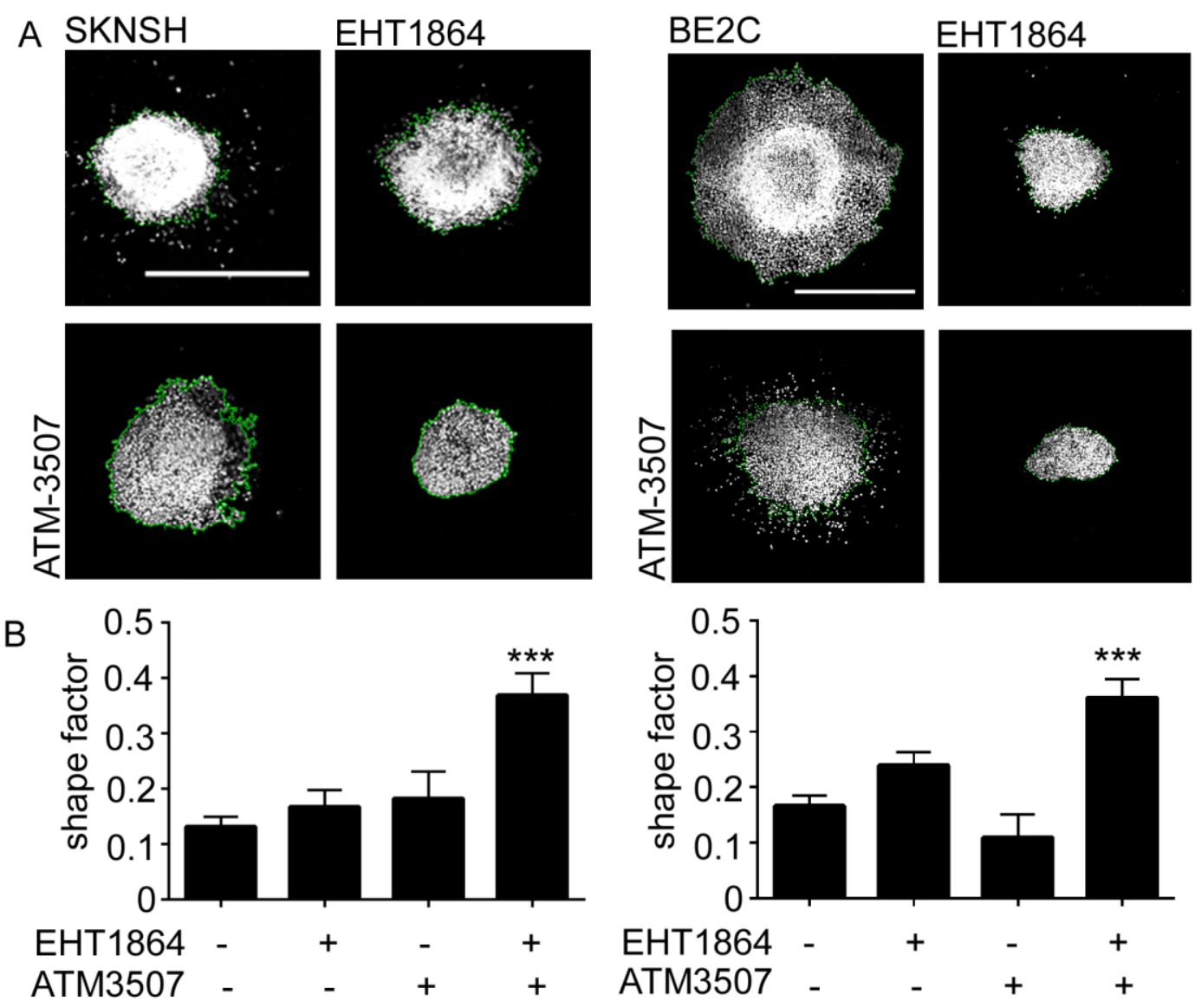




\section{A SKNSH \\ control}
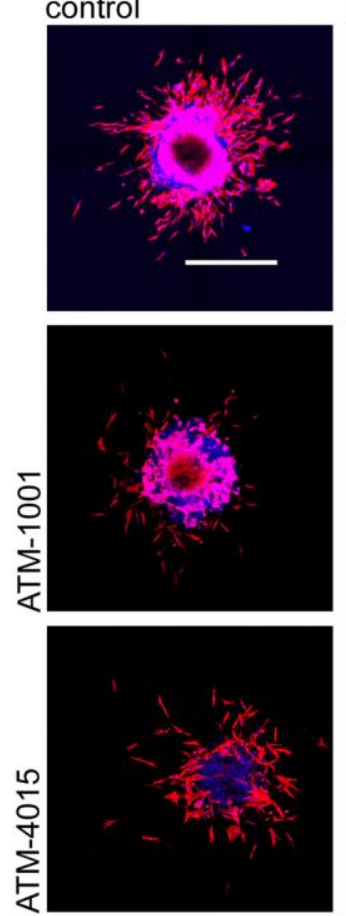

$C$ s

금

:

ATM-1001

ATM-4015

EHT1864
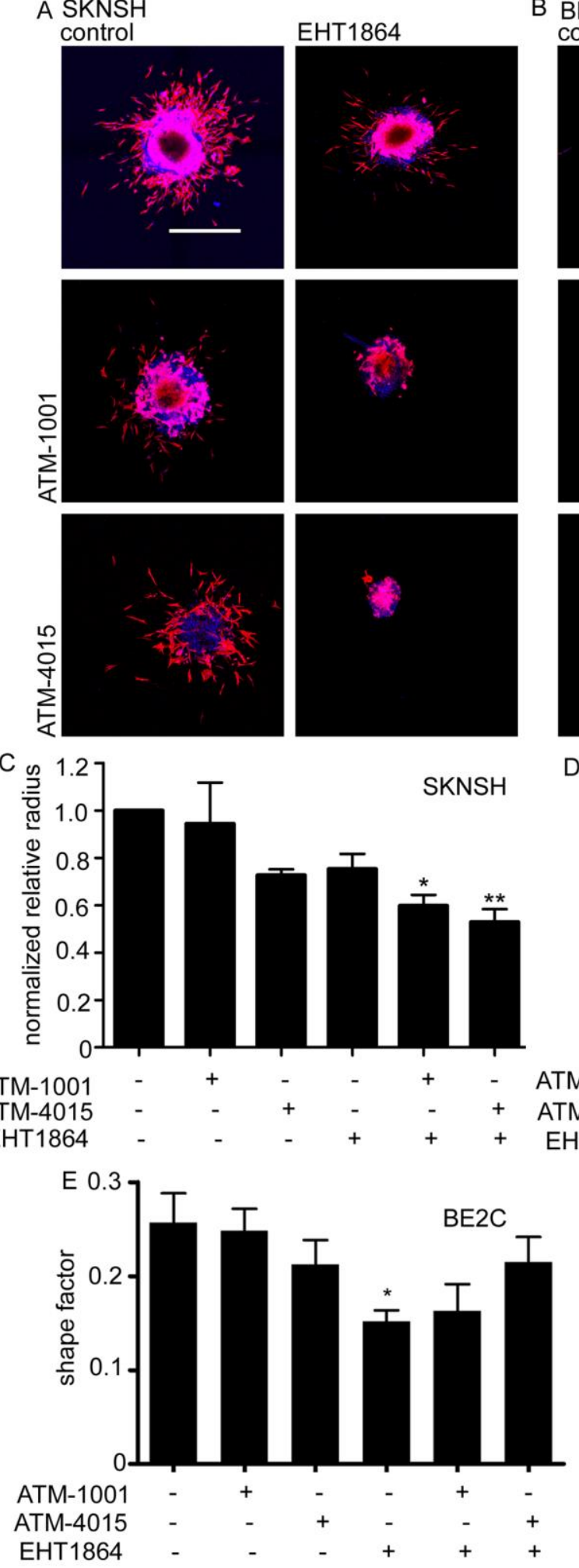

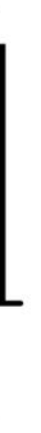

B BE2C

control

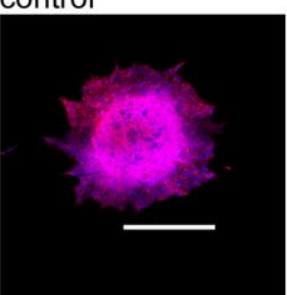

EHT1864
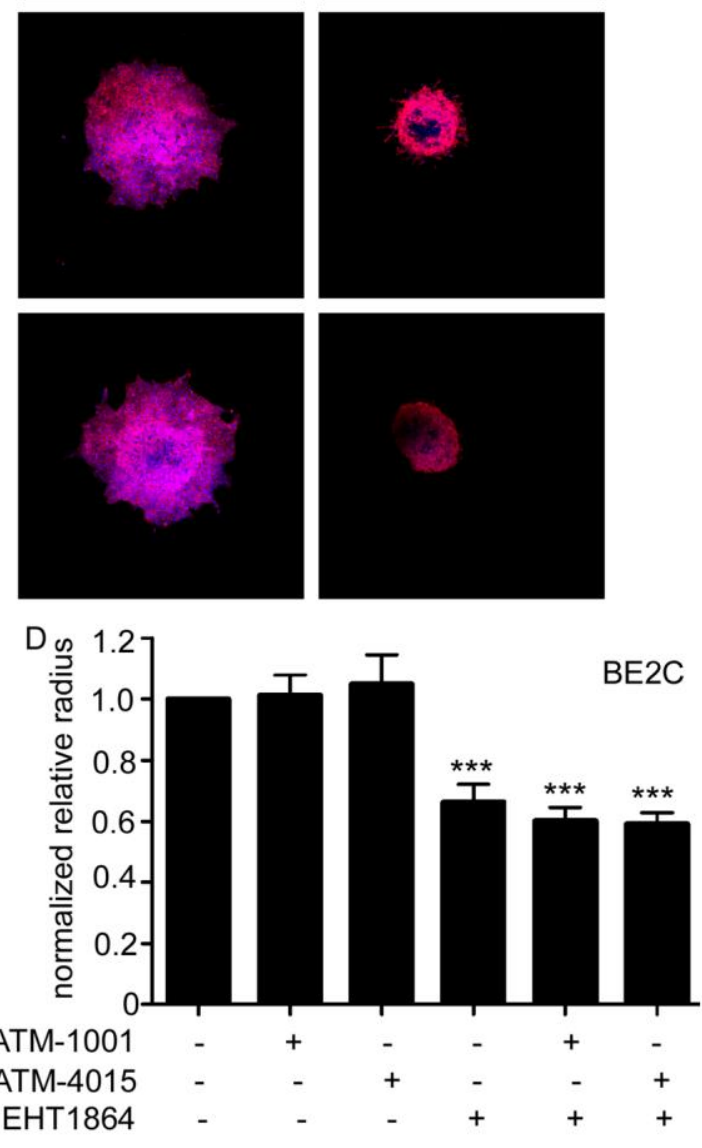

This article is protected by copyright. All rights reserved. 


\section{University Library}

\section{- M M I N E R VA \\ A gateway to Melbourne's research publications}

Minerva Access is the Institutional Repository of The University of Melbourne

Author/s:

Mitchell, CB;Stehn, JR;O'Neill, GM

Title:

Small molecule targeting of the actin associating protein tropomyosin Tpm3.1 increases neuroblastoma cell response to inhibition of Rac-mediated multicellular invasion

Date:

2018-07-01

\section{Citation:}

Mitchell, C. B., Stehn, J. R. \& O'Neill, G. M. (2018). Small molecule targeting of the actin associating protein tropomyosin Tpm3.1 increases neuroblastoma cell response to inhibition of Rac-mediated multicellular invasion. CYTOSKELETON, 75 (7), pp.307-317. https:// doi.org/10.1002/cm.21452.

Persistent Link:

http://hdl.handle.net/11343/285165 